\title{
Spin susceptibility in bilayered cuprates: resonant magnetic excitations
}

\author{
Ilya Eremin ${ }^{1}$, Dirk K. Morr ${ }^{2,3}$, Andrey V. Chubukov ${ }^{4}$, Karl Bennemann ${ }^{2}$ \\ 1 Max-Planck Institut für Physik komplexer Systeme, D-01187 Dresden, \\ Germany and Institute für Mathematische und Theoretische Physik, \\ TU-Braunschweig, D-38106 Braunschweig, Germany \\ 2 Institute für Theoretische Physik, Freie Universität Berlin, D-14195, Berlin, Germany \\ 3 Department of Physics, University of Illinois at Chicago, Chicago, IL 60607 \\ 4 Department of Physics, University of Wisconsin - Madison, Madison, WI 53706
}

(Dated: September 14, 2018)

\begin{abstract}
We study the momentum and frequency dependence of the dynamical spin susceptibility in the superconducting state of bilayer cuprate superconductors. We show that there exists a resonance mode in the odd as well as the even channel of the spin susceptibility, with the even mode being located at higher energies than the odd mode. We demonstrate that this energy splitting between the two modes arises not only from a difference in the interaction, but also from a difference in the freefermion susceptibilities of the even and odd channels. Moreover, we show that the even resonance mode disperses downwards at deviations from $\mathbf{Q}=(\pi, \pi)$. In addition, we demonstrate that there exists a second branch of the even resonance, similar to the recently observed second branch (the $Q^{*}$-mode) of the odd resonance. Finally, we identify the origin of the qualitatively different doping dependence of the even and odd resonance. Our results suggest further experimental test that may finally resolve the long-standing question regarding the origin of the resonance peak.
\end{abstract}

PACS numbers: 71.10.Ca,74.20.Fg,74.25.Ha,74.72.-h

\section{INTRODUCTION}

Magnetic excitations in the high-temperature superconductors are of fundamental interest. While it is currently still a topic of intense debate whether a continuum of magnetic excitations is responsible for the occurrence of superconductivity in the cuprates, the feedback effect of $d_{x^{2}-y^{2}}$-wave superconductivity on the magnetic excitation spectrum has been well established in the context of the "resonance peak". This peak has been observed by inelastic neutron scattering (INS) experiments in three different families of the high-temperature superconductors $1,2,3$. The doping dependence of the peak frequency, $\Omega_{\text {res }}(\mathbf{Q})$, the downward dispersion of the resonance, which tracks the momentum dependence of the particle-hole continuum, and the emergence of a second resonance branch further away from $\mathbf{Q}$ are all consistent with the idea that the resonance peak is a particle-hole bound state (i.e. a spin exciton) below the particle-hole continuum. According to theory $\underline{\underline{4}}$, this excitonic resonance is a fundamental property of a $d_{x^{2}-y^{2}}$ superconductor. (For a review of other theoretical scenarios, see $\left.\operatorname{Refs}, \frac{5,6,7}{6}\right)$.

Recent INS experiments in overdoped $\mathrm{YBa}_{2} \mathrm{Cu}_{3} \mathrm{O}_{6+x}$ (YBCO) revealed the formation of two resonance modes that differ by their symmetry with respect to the exchange of adjacent copper oxide layers ${ }^{8.9}$. The original resonance mode observed in the bilayer cuprate possesses an odd (o) symmetry while the new one exhibits an even (e) symmetry. The frequency of the even mode is larger while its intensity is smaller than that of the odd mode. Moreover, while the doping dependence of the odd mode is non-monotonic and roughly follows $\Omega_{\text {res }}^{o} \sim 5 k_{B} T_{C} 10$, the frequency of the even mode increases monotonically with decreasing doping 11,12 .

The splitting in energy between odd and even resonances has been analyzed theoretically in the past within the random phase approximation (RPA $)^{13}$, and has been attributed to the difference in the strength of the residual interaction leading to the bound state. The larger the interaction, the more the resonance is shifted downwards from the lower edge of the particle-hole $(p h)$ continuum. How such a difference in the interaction can easily be seen in the $t-J$ model, where the interactions in the even and odd spin channels are given by

$$
J_{o, e}(\mathbf{q})=J_{\|}(\mathbf{q}) \pm J_{\perp}
$$

with $J_{\|}, J_{\perp}>0$ being the in-plane and out-of-plane exchange interaction, respectively. Thus $J_{o}>J_{e}$, and the odd resonance occurs at a lower energy than the even one. Moreover, since the even mode lies closer to the $p h$ continuum its intensity is lower than that of the odd one. These two theoretical results $13,14,15$ are in good agreement with the experimental observations $8,9,11,12$.

In this article, we address three issues which have not yet been considered in earlier studies on the spin resonance in bilayer systems. First, we argue that the difference between the even and odd modes comes from two factors. One is the difference in the interaction, which was taken into account in earlier studies, another is the difference in the free-fermion susceptibilities of the even and odd channels which so far has been neglected. We show that the two factors are generally comparable to each other and depend on the same combination of parameters. Numerically, the difference in the interactions leads to a larger splitting between the even and odd resonances than the difference between the even and odd freefermion susceptibilities. Second, we extend our previous analysis of the odd resonance's dispersion ${ }^{16}$ to the even 
channel, and show that the even resonance mode also disperses downwards at deviations from Q. Moreover, we show that the downward dispersion of the even mode is more parabolic than that of the odd channel. Third, we demonstrate that there exists a second branch of the even resonance, similar to the recently observed second branch (the $Q^{*}$-mode $\frac{16}{}$ ) of the odd resonance, $\frac{17.18}{16}$. We show, following the approach of Ref $\frac{16}{16}$, that in the even channel, this second branch is much narrower in energy than in the odd one. These results suggest further experimental test that may finally resolve the long-standing question regarding the origin of the resonance peak.

Finally, we analyze the doping dependence of the even and odd resonances. In the overdoped region, both modes decrease due to a decreasing superconducting gap. In the opposite limit of zero doping even and odd resonances very likely evolve into the acoustic and optical spin wave modes of the bilayer Heisenberg antiferromagnet. We show, however, that, while plausible, the crossover from one regime to the other cannot be obtained within a simple RPA scheme chiefly because of the incorrect doping dependence of the free-fermion susceptibilities: the real part of both, the even and odd susceptibility decreases with decreasing doping at half-filling ${ }^{19}$. This behavior is a direct consequence of the fact that the even susceptibility diverges at the van-Hove singularity, and the odd susceptibility possesses a maximum near the van-Hove point.

The rest of the paper is organized as follows. In Sec.II we introduce our theoretical model and discuss the origin of the splitting between the even and odd resonance at $\mathbf{Q}=(\pi, \pi)$. In Sec. III we present the dispersion of the two resonances away from $\mathbf{Q}$ and show that a $Q^{*}$-mode also arises in the even channel. In Sec. IV we discuss the doping dependence of the resonances. Finally, in Sec. V we summarize our results and conclusions.

\section{EVEN AND ODD RESONANCES AT $\mathbf{Q}=(\pi, \pi)$}

The coupling between two $\mathrm{CuO}_{2}$-planes in a unit cell of YBCO is described by the interlayer hopping matrix element $t_{\perp}(\mathbf{k})=\frac{1}{4} t_{\perp}\left(\cos k_{x}-\cos k_{y}\right)^{2}$, Ref. 20. This coupling leads to the formation of bonding $(b)$ and antibonding (a) energy bands whose dispersion are given by

$$
\begin{aligned}
\varepsilon_{\mathbf{k}}^{a, b}= & -2 t\left(\cos k_{x}+\cos k_{y}\right)+4 t^{\prime} \cos k_{x} \cos k_{y} \\
& \pm \frac{1}{4} t_{\perp}\left(\cos k_{x}-\cos k_{y}\right)^{2}-\mu
\end{aligned}
$$

with $t=250 \mathrm{meV}, t^{\prime} / t=0.4, t_{\perp} / t=0.2$, and $\mu$ being the chemical potential (these parameters provide a good fit to the Fermi surface of the bilayered $\mathrm{Bi}_{2} \mathrm{Sr}_{2} \mathrm{CaCu}_{2} \mathrm{O}_{8+\delta}$ $\left.(\mathrm{BSCCO})^{21}\right)$. The resulting Fermi surfaces for the bonding and antibonding bands are shown in Fig. 1.

The bonding and antibonding creation and annihilation operators are related to the fermionic operators, $c_{1,2}$ in the two layers via

$$
c_{a}=\frac{c_{1}+c_{2}}{\sqrt{2}}, \quad c_{b}=\frac{c_{1}-c_{2}}{\sqrt{2}},
$$

It is also convenient to introduce even and odd components of the spins at site $\mathbf{i}$, which are given by

$$
\begin{aligned}
\mathbf{S}_{e}(\mathbf{i}) & =\frac{\mathbf{S}_{1}(\mathbf{i})+\mathbf{S}_{2}(\mathbf{i})}{2} \\
& =\frac{1}{2}\left(c_{a, \alpha}^{\dagger}(\mathbf{i}) \sigma_{\alpha, \beta} c_{a, \beta}(\mathbf{i})+c_{b, \alpha}^{\dagger}(\mathbf{i}) \sigma_{\alpha, \beta} c_{b, \beta}(\mathbf{i})\right), \\
\mathbf{S}_{o}(\mathbf{i}) & =\frac{\mathbf{S}_{1}(\mathbf{i})-\mathbf{S}_{2}(\mathbf{i})}{2} \\
& =\frac{1}{2}\left(c_{a, \alpha}^{\dagger}(\mathbf{i}) \sigma_{\alpha, \beta} c_{b, \beta}(\mathbf{i})+c_{b, \alpha}^{\dagger}(\mathbf{i}) \sigma_{\alpha, \beta} c_{a, \beta}(\mathbf{i})\right) .
\end{aligned}
$$

The experimentally measured susceptibility is related to the even and odd susceptibilities, $\chi^{e}=<S_{e} S_{e}>$ and $\chi^{o}=<S_{o} S_{o}>\operatorname{via}^{22}$

$$
\chi(\mathbf{q}, \omega)=\chi^{e}(\mathbf{q}, \omega) \cos ^{2} \frac{q_{z} d}{2}+\chi^{o}(\mathbf{q}, \omega) \sin ^{2} \frac{q_{z} d}{2},
$$

where $d$ is the separation between the layers. For noninteracting electrons, the susceptibilities in the even and odd channels are given by $\chi_{0}^{e}=\chi_{0}^{a a}+\chi_{0}^{b b}$ and $\chi_{0}^{o}=$ $\chi_{0}^{a b}+\chi_{0}^{b a}$, respectively, where $\chi_{0}^{a a}$ and $\chi_{0}^{b b}$ represent intraband particle-hole excitations, and $\chi_{0}^{a b}$ and $\chi_{0}^{b a}$ represent interband excitations. The free-fermion susceptibilities in the superconducting state at $T=0$ are given by $\underline{4,23}$

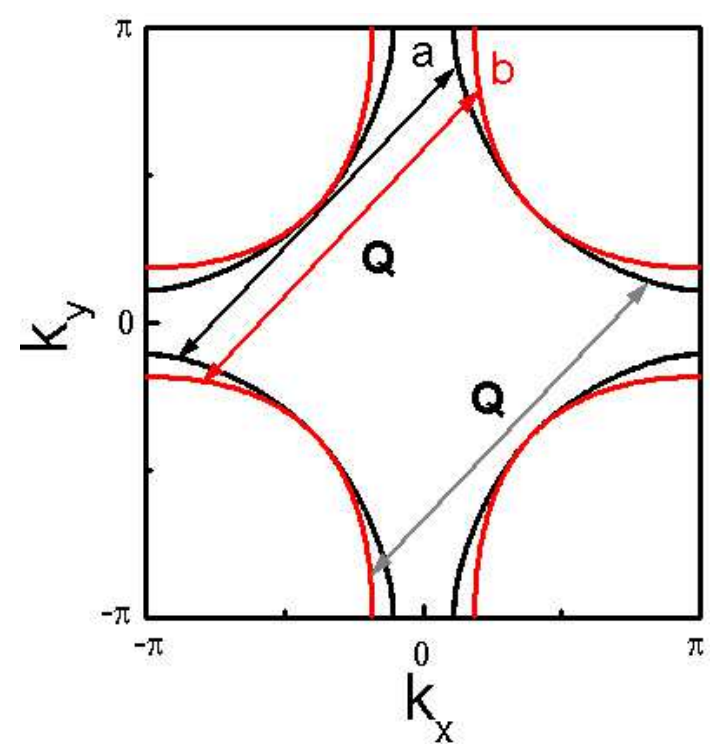

FIG. 1: (color online) Calculated Fermi surface(FS) for the bilayered cuprates as obtained from Eq.(2). The arrows indicates the transition between bonding-bonding $(b b)$, antibonding-antibonding $(a a)$ and antibonding-bonding ( $a b$, $b a)$ states for antiferromagnetic wave vector $\mathbf{Q}=(\pi, \pi)$. 


$$
\chi_{0}^{i j}(\mathbf{q}, \omega)=\frac{1}{4} \sum_{\mathbf{k}}\left(1-\frac{\varepsilon_{\mathbf{k}}^{i} \varepsilon_{\mathbf{k}+\mathbf{q}}^{j}+\Delta_{\mathbf{k}}^{i} \Delta_{\mathbf{k}+\mathbf{q}}^{j}}{E_{\mathbf{k}}^{i} E_{\mathbf{k}+\mathbf{q}}^{j}}\right)\left(\frac{1}{\omega+E_{\mathbf{k}+\mathbf{q}}^{j}+E_{\mathbf{k}}^{i}+i \Gamma}-\frac{1}{\omega-E_{\mathbf{k}+\mathbf{q}}^{j}-E_{\mathbf{k}}^{i}+i \Gamma}\right)
$$

with $i, j=a, b, E_{\mathbf{k}}^{i}=\sqrt{\left(\varepsilon_{\mathbf{k}}^{i}\right)^{2}+\left(\Delta_{\mathbf{k}}^{i}\right)^{2}}$, and $\Delta_{\mathbf{k}}^{i}$ is the superconducting gap in the bonding $(i=b)$ and antibonding $(i=a)$ band. In the following we assume that the pairing part of the Hamiltonian is symmetric with respect to the bilayers and given by

$$
\begin{aligned}
\mathcal{H}_{p p}= & \sum_{\mathbf{k}} \Delta(\mathbf{k})\left(c_{1, \uparrow}^{\dagger}(\mathbf{k}) c_{1, \downarrow}^{\dagger}(-\mathbf{k})+c_{2, \uparrow}^{\dagger}(\mathbf{k}) c_{2, \downarrow}^{\dagger}(-\mathbf{k})\right. \\
& + \text { h.c. }) \\
= & \sum_{\mathbf{k}} \Delta(\mathbf{k})\left(c_{a, \uparrow}^{\dagger}(\mathbf{k}) c_{a, \downarrow}^{\dagger}(-\mathbf{k})+c_{b, \uparrow}^{\dagger}(\mathbf{k}) c_{b, \downarrow}^{\dagger}(-\mathbf{k})\right. \\
& + \text { h.c. }),
\end{aligned}
$$

where $\Delta(\mathbf{k})=\frac{\Delta_{0}}{2}\left(\cos k_{x}-\cos k_{y}\right)$. It then follows that the pairing gap is the same for bonding and antibonding bands, implying $\Delta_{\mathbf{k}}^{a}=\Delta_{\mathbf{k}}^{b}=\Delta_{\mathbf{k}}$. However, the respective Fermi surfaces in both bands are located at different momenta $\mathbf{k}$ in the Brillouin zone. In order to obtain the full $\chi^{e, o}$, we use the random-phase approximation (RPA). Within RPA the even and odd parts of the full spin susceptibility are given by

$$
\chi_{R P A}^{\alpha}(\mathbf{q}, \omega)=\frac{\chi_{0}^{\alpha}(\mathbf{q}, \omega)}{1-g_{\alpha}(\mathbf{q}) \chi_{0}^{\alpha}(\mathbf{q}, \omega)},
$$

where $\alpha=o, e$ and $g_{e, o}(\mathbf{q})$ are the fermionic interaction vertices in the even and odd channels.

We first consider the spin susceptibility at momenta close to $\mathbf{Q}=(\pi, \pi)$. The dominant contribution to the susceptibilities comes from fermions near the hot spots, where both $\mathbf{k}$ and $\mathbf{k}+\mathbf{Q}$ are close to the Fermi surface. In a $d_{x^{2}-y^{2}}$-wave superconductor with the above $\Delta(\mathbf{k})$ one has $\Delta(\mathbf{k}+\mathbf{Q})=-\Delta(\mathbf{k})$. As a consequence, $\operatorname{Im} \chi_{0}^{e, o}$ exhibits discontinuities due the opening of the superconducting gap $\stackrel{24}{4}$. For the odd susceptibility, $\operatorname{Im} \chi_{0}^{a b}$ and $\operatorname{Im} \chi_{0}^{b a}$ exhibit a single discontinuity at $\Omega_{c}^{a b}(\mathbf{Q})=\left|\Delta_{\mathbf{k}}^{a}\right|+\left|\Delta_{\mathbf{k}+\mathbf{Q}}^{b}\right|$, where $\mathbf{k}$ is chosen such that $\varepsilon_{a}(\mathbf{k})=\varepsilon_{b}(\mathbf{k}+\mathbf{Q})=0$ (see Fig (1). Below this frequency, $\operatorname{Im} \chi_{0}^{a b}=0($ at $T=0)$. At the same time, $\operatorname{Im} \chi_{0}^{e}$ possesses two discontinuities located at $\Omega_{c}^{a a}(\mathbf{Q})=\left|\Delta_{\mathbf{k}}^{a}\right|+\left|\Delta_{\mathbf{k}+\mathbf{Q}}^{a}\right|$ and $\Omega_{c}^{b b}(\mathbf{Q})=\left|\Delta_{\mathbf{k}}^{b}\right|+\left|\Delta_{\mathbf{k}+\mathbf{Q}}^{b}\right|$, where $\mathbf{k}$ is again chosen such that both fermions are at the Fermi surface (see Fig. 1). $\operatorname{Im} \chi_{0}^{e}$ is zero below the lower discontinuity, and jumps between two finite values at the higher discontinuity. Analyzing Eq. (6), we find $\Omega_{c}^{b b}(\mathbf{Q})<\Omega_{c}^{a b}(\mathbf{Q})<\Omega_{c}^{a a}(\mathbf{Q})$.

Hence, in the even and odd channel, the susceptibility at low frequencies is purely real and, according to Eq.(66), one finds that the bare $\chi_{0}^{\alpha}(\mathbf{Q}, \omega)(\alpha=a, b)$ behaves as

$$
\chi_{0}^{\alpha}(\mathbf{Q}, \omega)=\chi_{0}^{\alpha}(\mathbf{Q}, 0)-A_{\alpha} f\left(\omega / \Omega_{i j}\right),
$$

where $A_{\alpha}>0$, and $f(x) \propto x^{2}$ at small $x$, and $f(x) \sim$ $|\log (1-x)|$ near $x=1$. Substituting this result in Eq. (8), one finds that since $f(x)$ changes between 0 and $\infty$ when $x$ changes between 0 and 1 , the susceptibilities in both the odd and even channel develop resonances below the thresholds of the particle-hole continuum, at frequencies $\Omega_{e, o}$ where $1=g_{e, o} \chi_{0}^{e, o}\left(\mathbf{Q}, \Omega_{e, o}\right)$.

As mentioned above, there are two reasons why the resonances in the even and odd channels occur at different frequencies. One is that the even and odd freefermion susceptibilities, $\chi_{0}^{e, o}(\mathbf{Q}, \omega)$ are different, another one is that the interactions are different in the even and odd channels. The difference between the resonance frequencies in the even and odd channels due to the difference in $\chi_{0}^{e, o}(\mathbf{Q}, \omega)$ arises predominantly from the fact that the (dimensionless) magnetic correlation lengths $\xi_{e, o}^{-2}=1-g_{e, o} \chi_{0}^{e, o}(\mathbf{Q}, 0)$ are different in both channels already in the normal state. Additional differences between $\chi_{0}^{e, o}(\mathbf{Q}, 0)$ which arise in the superconducting state scale as $\Delta_{0} / E_{F}$, and are hence small and can be neglected. Assuming that the difference between the even and the odd resonance is small, and that the resonance frequencies are sufficiently low, such that $f(x)$ in Eq.(9) scales as $x^{2}$, we find after some simple algebra that

$$
\frac{\Omega_{e}-\Omega_{o}}{\Omega_{o}}=\frac{\xi_{e}^{-1}-\xi_{o}^{-1}}{\xi_{o}^{-1}}
$$

The r.h.s. of the above equation is in turn related to the difference in the normal state static $\chi$ via

$$
\frac{\xi_{e}^{-1}-\xi_{o}^{-1}}{\xi_{o}^{-1}}=\frac{g}{2} \xi_{o}^{2}\left(\chi_{0}^{a b}+\chi_{0}^{b a}-\chi_{0}^{a a}-\chi_{0}^{b b}\right) .
$$

The dominant contributions to the r.h.s. of Eq.(11) come from fermions in hot regions near $(0, \pi)$ and $(\pi, 0)$, for which the $t_{\perp}$ term in the dispersion, Eq.(2) reduces to $\pm t_{\perp}$. Expanding the r.h.s. of Eq.(11) to leading order in $t_{\perp}$, we obtain

$$
\frac{\xi_{e}^{-1}-\xi_{o}^{-1}}{\xi_{o}^{-1}}=t_{\perp}^{2} \frac{g \xi_{o}^{2}}{2 \pi^{3}} \int \frac{d \omega d^{2} k}{\left(\epsilon_{k}-i \omega\right)^{2}\left(\epsilon_{k+q}-i \omega\right)^{2}}
$$

where $\epsilon_{k}$ is the in-plane dispersion (i.e., Eq.(2) with $t_{\perp}=$ $0)$. Linearizing $\varepsilon_{\mathbf{k}}$ and $\varepsilon_{\mathbf{k}+\mathbf{Q}}$ in the hot regions as $v_{F}\left(k_{x}+\right.$ $\left.k_{y}\right) / \sqrt{2}$ and $v_{F}\left(k_{x}-k_{y}\right) / \sqrt{2}$, respectively, substituting this expansion into the susceptibilities, and performing the integration, we obtain

$$
\frac{\xi_{e}^{-1}-\xi_{o}^{-1}}{\xi_{o}^{-1}}=t_{\perp}^{2} \frac{8 g \xi_{o}^{2}}{\pi^{2} v_{F}^{3} k_{\max }}
$$

where $k_{\max } \sim k_{F}$ is the upper limit of the integration over momentum. Observe that the r.h.s. of Eq.(13) is 
positive, implying that because of the difference in the even and odd susceptibilities, the resonance in the even channel occurs at a larger frequency than the resonance in the odd channel. To estimate the strength of the effect, we use $v_{F} k_{F} \sim 0.5 \mathrm{eV} \sim 2 t, g=U / 4$, where $U$ us the Hubbard interaction (in the RPA, $m U / 2 \pi \approx 1$ ), set $k_{\max }=k_{F}$, and define $J_{\perp}=4 t_{\perp}^{2} / U, J=4 t^{2} / U$. We then have

$$
\frac{\xi_{e}^{-1}-\xi_{o}^{-1}}{\xi_{o}^{-1}}=\frac{\xi_{o}^{2}}{4 \pi} \frac{J_{\perp}}{J}
$$

The second source for the difference between $\Omega_{e}$ and $\Omega_{o}$ is the difference in the interaction strength between the two channels. As mentioned above, within the $t-J$ model, the two interactions are given by $J_{o, e}=J_{\|}(\mathbf{q}) \pm$ $J_{\perp}$. At $\mathbf{q}=\mathbf{Q}$, this effect alone leads to

$$
\frac{\xi_{e}^{-1}-\xi_{o}^{-1}}{\xi_{o}^{-1}}=\frac{J_{\perp}}{J},
$$

where $J=J_{\|}(\mathbf{q}=\mathbf{Q})$. We see that both effects described by Eqs. (14) and (15) are in fact of the same order, and both lead to a larger $\Omega_{e}$ compared to $\Omega_{o}$. Moreover, the effect of the $t_{\perp}$ dependence of the interaction is larger, at least near optimal doping, where $\xi_{o} \sim 1$. However, with decreasing doping, and hence increasing $\xi_{o}$, the role of the difference in the even and odd free-fermion susceptibilities may become more dominant.

In Fig. 2, we present the results for the bare and full susceptibilities at optimal doping, corresponding to $\mu=-1.195 t$, that were obtained from a numerical evaluation of Eqs.(6) and (8). We see that Re $\chi_{0}$ in even and

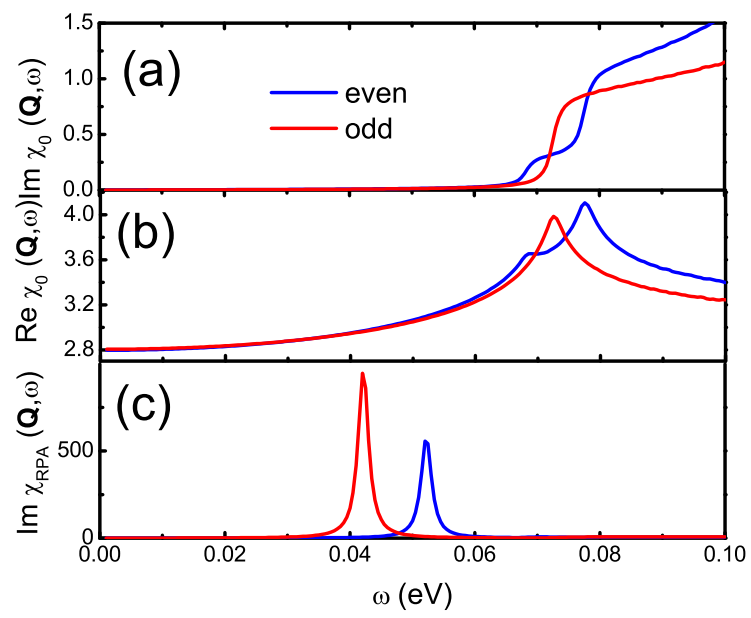

FIG. 2: (color online) (a) $\operatorname{Im} \chi_{0}^{e, o}$, (b) $\operatorname{Re} \chi_{0}^{e, o}$ and (c) $\operatorname{Im} \chi_{R P A}^{e, o}$ as a function of frequency at the antiferromagnetic wave vector $\mathbf{Q}=(\pi, \pi)$.

odd channels are almost identical below $2 \Delta_{0}$, i.e. the difference in the susceptibilities is too small to give rise to an observable difference between $\Omega_{e}$ and $\Omega_{o}$. This agrees with our analytic treatment. Hence, the difference between $\Omega_{e}$ and $\Omega_{o}$ arises from the difference in the effective interactions $g_{e}$ and $g_{o}$. To reproduce the experimentally measured frequency splitting between both resonances at $\mathbf{Q}$, and the dispersion of the two modes (see below), we use

$$
g_{o, e}(\mathbf{q})=J_{0}\left\{1-0.1\left[\cos \left(q_{x}\right)+\cos \left(q_{y}\right)\right]\right\} \pm 0.027 J_{0}
$$

According to Eq.(1), the first (second) term on the r.h.s. of the above equation can be interpreted as arising from the in-plane (out-of-plane) exchange interaction $J_{\|}(\mathbf{q})$ $\left(J_{\perp}\right)$.

We present the RPA susceptibilities $\chi_{R P A}^{e, o}$ at $\mathbf{Q}$ in Fig. 2(c). We see that both even and odd susceptibilities show resonance behavior. By construction, the resonance in the even channel occurs at a larger frequency than the odd resonance. Accordingly, the intensity of the even resonance is smaller, which agrees well with the experimental observations $\stackrel{8}{ }$.
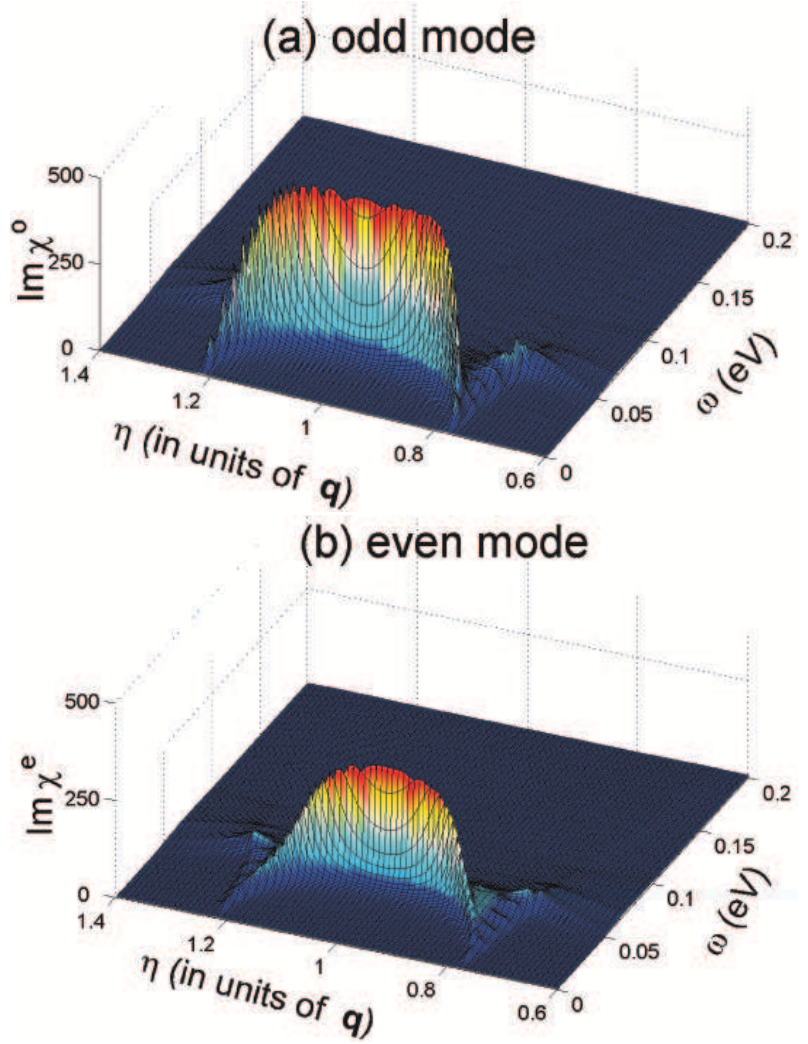

FIG. 3: (color online) RPA results for magnetic excitations in a bilayered $d_{x^{2}-y^{2}}$ superconductor at optimal doping. Calculated $\operatorname{Im} \chi^{o}(\mathrm{a})$ and $\operatorname{Im} \chi^{e}(\mathrm{~b})$ obtained from Eq. (8) as a function of momentum along $\mathbf{q}=\eta(\pi, \pi))$ and frequency in the SC state. 


\section{THE DISPERSION OF THE RESONANCE PEAK}

We next consider the dispersion of the even and the odd resonances and present in Fig. 3 a plot of $\operatorname{Im} \chi_{R P A}^{e, o}(\mathbf{q}, \Omega)$ at optimal doping along $\mathbf{q}=\eta(\pi, \pi)$ as a function of frequency. The momentum dependence of the odd mode's frequency and intensity, shown in Fig. 3(a), is quite similar to that of the resonance mode in the singlelayer model ${ }^{16}$. In particular, away from $\mathbf{Q}$ three discontinuities in $\operatorname{Im} \chi_{0}^{o}$ emerge, corresponding to scattering channels with momenta $\mathbf{q},(2 \pi, 0)-\mathbf{q}$, and $(2 \pi, 2 \pi)-\mathbf{q}$. The first momentum corresponds to a direct transition, while the last two momenta describe scattering processes involving Umklapp scattering ${ }^{16}$. As discussed before, the resonance can occur only at frequencies below the lowest discontinuity in $\operatorname{Im} \chi_{0}^{o 4,16}$. Since the superconducting gap decreases towards the diagonal of the Brillouin Zone (BZ), the resonance dispersion follows the momentum dependence of the $p h$ continuum, forming a parabolic-like shape ${ }^{4,16}$. Upon reaching $\mathbf{Q}_{0} \approx(0.8,0.8) \pi$ corresponding to the wave vector connecting the nodal points of the superconducting gap on the Fermi surface, the spin gap vanishes, and no resonance is possible. For even smaller $\mathbf{q}$ one finds that another resonance branch emerges, the so called $Q^{*}$ mode, arising from an umklapp transition ${ }^{16}$.

In contrast, the even part of the spin susceptibility exhibits six discontinuities in $\operatorname{Im} \chi_{0}^{e}$ away from $\mathbf{Q}=(\pi, \pi)$. Intraband scattering within the bonding and antibonding bands each gives rise to three of these discontinuities. Similarly to the odd susceptibility, we find that a genuine resonance occurs only below the lowest discontinuity in $\operatorname{Im} \chi_{0}^{b b}$ due to the direct transition with momentum q. This transition is again responsible for the paraboliclike shape of the even mode's dispersion, as shown in Fig. 3(b). However, we find that the intensity of the even resonance falls off much faster as one moves away from $\mathbf{Q}$ than that of the odd one. Since the superconducting gap and the splitting of the Fermi surfaces is zero along the diagonal of the BZ, the position of the so-called "silent band" is the same for the odd and for the even channel. Thus, both resonances merge together at $\mathbf{Q}_{0} \approx(0.8,0.8) \pi$ (see also Fig $\left.4(\mathrm{c})\right)$. Similar to the resonance in the odd channel, we find that for momenta smaller than $\mathbf{Q}_{0}$, an umklapp transition leads to the formation of a $Q^{*}$ mode in the even channel. However, its energy range is much smaller than that of the odd $Q^{*}$ mode due to the proximity to the $p h$ continuum. For the odd as well as the even resonance mode we find that while the intensity of the $Q$ mode (i.e., the mode originating at $\mathbf{Q})$ is largest along $\mathbf{q}=(\pi, \eta \pi)$ and $\mathbf{q}=(\eta \pi, \pi)$, the $Q^{*}$ mode has its largest intensity along the diagonal direction, i.e., along $\mathbf{q}=\eta(\pi, \pi)$ and $\mathbf{q}=[(2-\eta) \pi, \eta \pi]$. As previously discussed 16 , this rotation of the intensity pattern by $45^{\circ}$ reflects the qualitative difference in the origin of the first and second resonances.

\section{DOPING DEPENDENCE OF THE EVEN AND ODD RESONANCES}

Next, we consider the doping dependence of the resonance modes in the odd and even channels. In order to describe the doping dependence, it is necessary to know that of the superconducting gap as well as that of $g_{o, e}(\mathbf{q})$. The doping dependence of the superconducting gap, which is shown in Fig. 4(b), is taken from recent ARPES experiments ${ }^{25}$ which suggest that the superconducting gap increases by about $10 \div 20 \%$ going from the optimally doped to the underdoped cuprates. In order to

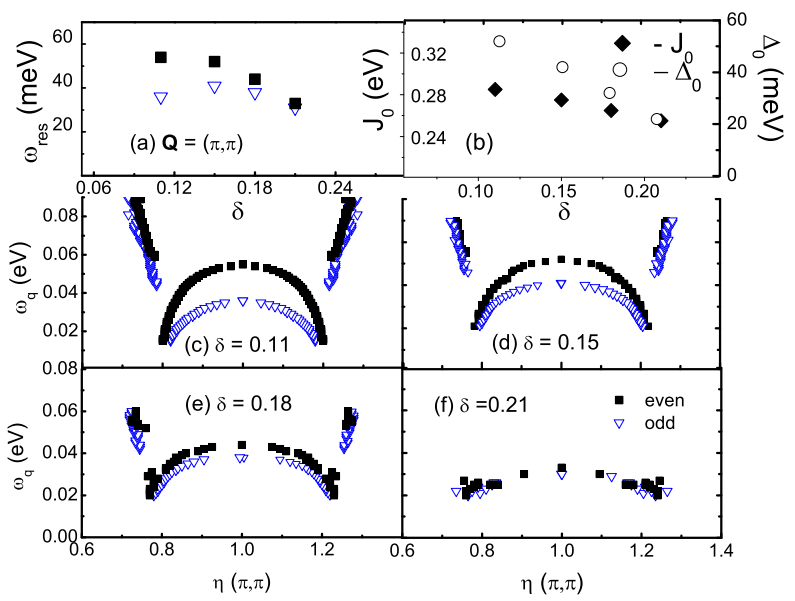

FIG. 4: (color online) (a) Doping dependence of (a) the resonance frequency at $\mathbf{Q}$ in the odd and even channel, and (b) the superconducting gap $\Delta_{0}$ and $J_{0}$. (c)-(f) Dispersion of the even and odd modes for various doping concentrations in the (c) underdoped, (d) optimally doped, and (e) and (f) overdoped regime.

describe the doping dependence of $g_{o, e}(\mathbf{q})$, we leave the momentum dependence of $g_{o, e}(\mathbf{q})$ unchanged, and only change the overall prefactor, $J_{0}$, in Eq.(16), as a function of doping, by fitting the frequency of the resonance in the odd channel. The doping dependence of $J_{0}$ is also shown in Fig. 4(b). We find that this procedure provides a satisfactory fit to the experimentally measured dispersion of both resonance modes over a considerable range of doping.

In Fig. 4(a) we present the doping dependence of the resonance in the even and odd channels at $\mathbf{Q}=(\pi, \pi)$. As expected from the discussions above, we find that with increasing doping, the energy splitting between both modes decreases, and for $\delta=0.21$ is only about $\Delta \omega_{\text {res }} \approx 1$ meV at $\mathbf{Q}$, while for $\delta=0.15$ one has $\Delta \omega_{\text {res }} \approx 12 \mathrm{meV}$. This decrease in the splitting is observed over the entire dispersion of the resonance modes in the even and odd channels, which we present in Figs. 4(c)-(f) for several different doping levels. In addition, we find that the dispersion of the even mode exhibits a continuous downshift 
with increasing doping, while that of the odd mode first shifts upwards with increasing doping in the underdoped systems, but shift downwards in the overdoped regime. In order to understand this qualitative difference between the underdoped and overdoped region, we note that in general, the doping dependence of the resonance modes is determined by that of the superconducting gap (which in turn determines that of the $p h$ continuum) as well as that of $g_{o, e}(\mathbf{q})$. While a decrease of the superconducting gap, and hence a downward shift in frequency of the $p h$ continuum leads to a downward shift of the resonances, a decrease of $g_{o, e}(\mathbf{q})$, in contrast, leads to an upward shift of the modes' dispersion.

Since the dispersion of the even resonance is located in frequency close to the $p h$ continuum, and $\operatorname{Re} \chi_{0}^{e}$ varies strongly in the vicinity of the $p h$ continuum due to its logarithmic singularity, it follows that the dispersion of the even resonance is rather insensitive to changes in $g_{e}(\mathbf{q})$. As a result, the doping dependence of the even resonance is predominantly determined by that of the ph continuum, exhibiting a continuous downward shift in energy with increasing doping. In contrast, in the underdoped regime, the energy difference between the $p h$ continuum and the odd mode's dispersion is rather large, and $\operatorname{Re} \chi_{0}^{o}$ varies only weakly around the resonance frequency. As a result, the resonance frequency is very sensitive to changes in $g_{o}(\mathbf{q})$. Therefore, it is the decrease in $g_{o}(\mathbf{q})$ with increasing doping (and not the decrease in the superconducting gap) that determines the doping dependence of the odd mode's dispersion and leads to its upward shift in energy in the underdoped regime. Around optimal doping, the odd mode's dispersion has become sufficiently close to the $p h$ continuum, that the mode's further doping dependence is now determined by that of the $p h$ continuum, and not any longer by that of $g_{o}(\mathbf{q})$, similar to the case of the even mode. Hence, the two opposite effects arising from a decrease of the superconducting gap and that of $g_{o}(\mathbf{q})$ lead to the qualitatively different doping dependence of the odd mode's dispersion in the underdoped and overdoped regime. Note that with increasing doping, and the resulting downward shift of the $p h$ continuum, the momentum range over which the $Q^{*}$-mode can be observed, decreases.

Finally, we briefly discuss the doping dependence of $\chi^{e, o}(\mathbf{Q}, \omega=0)$. If indeed, the suggested above, the odd and even resonance are transformed into the acoustic and optical branches of the spin wave dispersion in the antiferromagnetically ordered phase, one would expect that $\chi_{0}^{o}(\mathbf{Q}, \omega=0)$ increases with decreasing doping. As a result, one would see a downward shift in the odd mode's dispersion even for a doping independent $J_{0}$. One finds, however, that the doping dependence of $\chi_{0}^{o}(\mathbf{Q}, 0)$, which is obtained from Eq.(6) by simply changing the chemical potential, $\mu$, defies this expectation. This is shown in Fig 5, where we present the doping dependence of $\chi_{0}^{o, e}(\mathbf{Q}, 0)$. Note that the even susceptibility possesses two logarithmic divergences as a function of doping which occur when either the bonding or antibonding Fermi

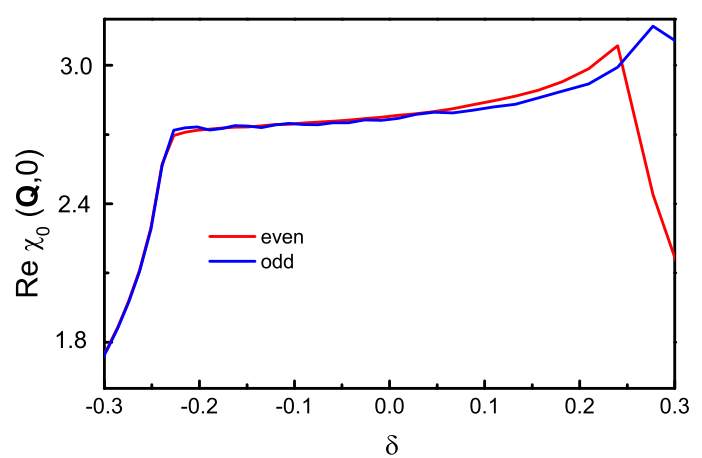

FIG. 5: (color online) $\operatorname{Re} \chi_{0}^{e, o}(\mathbf{Q}, 0)$ as a function of doping concentration in the normal state.

surfaces touches the van Hove $(\mathrm{vH})$ points $( \pm \pi, 0)$ and $(0, \pm \pi)$ and undergo a topological transition from a holelike to and electron-like Fermi surface. These transitions occur at a doping level of $x \approx 0.23$ for the antibonding band and at $x \approx 0.55$ for the bonding band (not shown). In contrast, the odd susceptibility, which arises from scattering transitions between the bonding and antibonding bands does not exhibit a logarithmic divergence, but is simply enhanced and exhibits a finite maximum. If we define the minimum distance (in momentum space) of the bonding and antibonding Fermi surfaces to the $\mathrm{vH}$ point $(0, \pi)$ by $k_{a}(\mu)$ and $k_{b}(\mu)$, respectively, then $\operatorname{Re} \chi_{0}^{o}(\mathbf{Q}, 0)$ exhibits a maximum at that doping level for which the smaller of $k_{a}(\mu)$ and $k_{b}(\mu)$ possesses a maximum. Defining $k_{\min }(\mu)=\min \left[k_{a}(\mu), k_{b}(\mu)\right]$ one finds

$$
\operatorname{Re} \chi^{o}(\mathbf{Q}, 0) \sim \text { const. }+\frac{1}{2 \pi t} \arcsin \left[k_{\text {min }}^{2}(\mu) \frac{t}{t_{\perp}}\right]
$$

Note that for doping levels below that at which the van Hove singularity in $\operatorname{Re}_{0}^{e}(\mathbf{Q}, 0)$ or the maximum in $\operatorname{Re} \chi_{0}^{o}(\mathbf{Q}, 0)$ occurs, the susceptibilities decrease monotonically with decreasing doping, as shown in Fig. 5. This doping dependence clearly reflects a shortcoming of the weak-coupling approach used above, which fails to capture the strong correlation effects that are not only responsible for the occurrence of antiferromagnetism, but are very likely also the key ingredients in the explanation of the pseudo-gap region in the underdoped cuprates. It is interesting to note in this context that recent studies of the doping dependence of $\chi_{0}(\mathbf{Q}, 0)$ for a single layer system within the FLEX approach find that the vH singularity is eliminated by interaction effects, and that starting from the overdoped region $\chi_{0}(\mathbf{Q}, 0)$ increases monotonically with decreasing doping ${ }^{26}$. This shortcoming of the approach used above is effectively compensated by a phenomenologically introduced doping dependence of $g^{e, o}$ which increases with decreasing doping. This phenomenological approach, however, does not allows us to fully explain the doping dependence of the resonant excitations in the underdoped cuprates. In particular, it 
leaves open the question how the downward dispersion of the resonance mode observed in the optimally doped cuprates is transformed into the upward dispersion of the acoustic spin-wave branch.

\section{SUMMARY}

In this study, we have investigated the form of magnetic resonance excitations in the even and odd spin channel of the bilayer cuprates in the superconducting state. We obtain a number of new results suggesting further experimental test that may finally resolve the longstanding question concerning the origin of the resonance peak. First, we show that the energy splitting between the even and odd resonances arises not only from a different interaction strength in both channels, but also from a the difference in the free-fermion susceptibilities in the even and odd channels. Both effects scale as $\sim J_{\perp} / J$ and lead to a frequency for the even resonance that is larger than that of the odd resonance. However, at least at optimal doping, the numerical prefactors are such that the energy splitting is dominated by the difference in the interaction strength and not by the difference in the freefermion susceptibilities. Since the latter scales with $\xi_{o}^{2}$, the relative importance of these two effects might change in the underdoped cuprates. In agreement with previous results $13,14,15$ we also find that the intensity of the even resonance is weaker than that of the odd resonance. Second, we computed the dispersion of the even resonance and showed that the even resonance also disperses downward as one moves away from $\mathbf{Q}=(\pi, \pi)$. Moreover, we demonstrated that the downward dispersion of the even mode is more parabolic than that of the odd channel. Third, we showed that there exists a second branch of the even resonance, similar to the recently observed second branch (the $Q^{*}$-mode ${ }^{16}$ ) of the odd resonance, 17,18 . We find, however, that in the even channel, this second branch is much narrower in energy than in the odd one. Fourth, we studied the doping dependence of the both resonance modes, and find that that of the even mode is determined by the downward shift of the $p h$ continuum with increasing doping. In contrast, the upward shift in frequency of the odd resonance in the underdoped cuprates is determined by the decrease in $g_{o}$ with increasing doping, while in the overdoped regime, the odd resonance follows the doping dependence of the $p h$ continuum. Our results demonstrate that the structure of magnetic excitations in the superconducting state of the bilayered cuprates is dominated by the topology of the Fermi surface, the interaction strength in the even

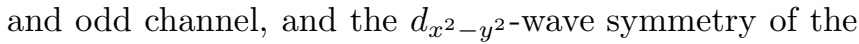
superconducting gap.

Finally, we note that the experimental situation has recently been complicated by the report that an even resonance exists at incommensurate wave vectors only $\underline{\underline{9}}$. This result contradicts earlier studies which have found that the even resonance exhibits the largest intensity at $\mathbf{Q}=(\pi, \pi)^{11}$. The origin of this experimental discrepancy is currently unclear.

We thank Y. Sidis, Ph. Bourges, P. Dai, and I.A. Larionov for helpful discussions. The present work is supported by the DAAD Collaborative German-US research grant No. D/05/50420. D.K.M. acknowledges financial support by the Alexander von Humboldt Foundation, the National Science Foundation under Grant No. DMR-0513415 and the U.S. Department of Energy under Award No. DE-FG02-05ER46225. A.C. acknowledges support from NSF DMR 0604406.
1 J. Rossat-Mignod, L. P. Regnault, C. Vettier, P. Bourges, P. Burlet, J. Bossy, J. Y. Henry and G. Lapertot, Physica C 185-189, 86 (1991); H.F. Fong, P. Bourges, Y. Sidis, L.P. Regnault, J. Bossy, A. Ivanov, D.L. Milius, I.A. Aksay, and B. Keimer, Phys. Rev. B 61, 14773 (2000); P. Dai, H. A. Mook, R. D. Hunt, F. Dogan, Phys. Rev. B 63, 054525 (2001).

2 H.F. Fong, P. Bourges, Y. Sidis, L.P. Regnault, A. Ivanov, G.D. Gu, N. Koshizuka, and B. Keimer, Nature (London) 398, 588 (1999).

3 H. He, P. Bourges, Y. Sidis, C. Ulrich, L.P. Regnault, S. Pailhes, N.S. Berzigiarova, N.N. Kolesnikov, and B. Keimer, Science 295, 1045 (2002).

4 H.F. Fong, B. Keimer, P.W. Anderson, D. Reznik, F. Dogan, and I.A. Aksay Phys. Rev. Lett. 75, 316 (1995); Ar. Abanov and A.V. Chubukov, Phys. Rev. Lett. 83, 1652 (1999); J. Brinckmann and P. A. Lee, Phys. Rev. Lett. 82, 2915 (1999); Y.-J. Kao, Q. Si, and K. Levin, Phys. Rev. B 61, 11898(R) (2000); F. Onufrieva and P. Pfeuty, Phys. Rev. B 65, 054515 (2002); D. Manske, I. Eremin, and K. H. Bennemann, Phys. Rev. B 63, 054517 (2001); M.R.
Norman, Phys. Rev. B 61, 14751 (2000); ibid 63, 092509 (2001); A. Chubukov, B. Janko and O. Tchernyshov, Phys. Rev. B 63, 180507(R) (2001); I. Sega, P. Prelovek, and J. Bonca, Phys. Rev. B 68, 054524 (2003).

5 L. Yin, S. Chakravarty, and P.W. Anderson, Phys. Rev. Lett. 78, 3559 (1997); E. Demler and S.C. Zhang, Phys. Rev. Lett. 75, 4126 (1995); D.K. Morr and D. Pines, Phys. Rev. Lett. 81, 1086 (1998).

${ }^{6}$ M. Vojta and T. Ulbricht, Phys. Rev. Lett. 93, 127002 (2004); G.S. Uhrig, K.P. Schmidt, and M. Grüninger, Phys. Rev. Lett. 93, 267003 (2004); F. Krüger and S. Scheidl, Phys. Rev. B 70, 064421 (2004); G.S. Uhrig, K.P. Schmidt, and M. Grüninger, J. Phys. Soc. Jpn. (Suppl.) 74, 86 (2005); M. Vojta, T. Vojta, and R.K. Kaul, Phys. Rev. Lett. 97, 097001 (2006).

7 G. Seibold, and J. Lorenzana Phys. Rev. B 73, 144515 (2006); ibid. Phys. Rev. Lett. 94, 107006 (2005).

8 S. Pailhes, Y. Sidis, P. Bourges, C. Ulrich, V. Hinkov, L.P. Regnault, A. Ivanov, B. Liang, C.T. Lin, C. Bernhard, and B. Keimer, Phys. Rev. Lett. 91, 237002 (2003).

9 H. Woo, P. Dai, S.M. Hayden, H.A. Mook, T. Dahm, D.J. 
Scalapino, T.G. Perring, and F. Dogan, Nature Physics (London) 2, 600 (2006).

10 H.F. He, Y. Sidis, P. Bourges, G.D. Gu, A. Ivanov, N. Koshizuka, B. Liang, C.T. Lin, L.P. Regnault, E. Schoenherr, and B. Keimer, Phys. Rev. Lett. 86, 1610 (2001).

11 S. Pailhes, C. Ulrich, B. Fauque, V. Hinkov, Y. Sidis, A. Ivanov, C.T. Lin, B. Keimer, and P. Bourges, Phys. Rev. Lett. 96, 257001 (2006).

12 L. Capogna, B. Fauque, Y. Sidis, C. Ulrich, Ph. Bourges, S. Pailhes, A. Ivanov, J.L. Tallon, B. Liang, C.T. Lin, A.I. Rykov, and B. Keimer, cond-mat/0610869 (unpublished).

13 A.J. Millis and H. Monien, Phys. Rev. B 54, 16172 (1996).

14 J. Brinckmann and P.A. Lee, Phys. Rev. B 65, 014502 (2001); T. Li, Phys. Rev. B 64, 012503 (2001).

15 H. Yamase, and W. Metzner, Phys. Rev. B 73, 214517 (2006).

16 I. Eremin, D.K. Morr, A.V. Chubukov, K.H. Bennemann, and M.R. Norman, Phys. Rev. Lett. 94, 047001 (2005).

17 S. Pailhes, Y. Sidis, P. Bourges, V. Hinkov, A. Ivanov, C. Ulrich, L.P. Regnault, and B. Keimer, Phys. Rev. Lett. 93, 167001 (2004); D. Reznik, P. Bourges, L. Pintschovius, Y. Endoh, Y. Sidis, T. Masui, and S. Tajima, Phys. Rev. Lett. 93, 207003 (2004).
18 S.M. Hayden, H.A. Mook, P.C. Dai, T.G. Perring, and F. Dogan, Nature (London) 429, 531 (2004).

19 F. Onufrieva and P. Pfeuty, Phys. Rev. Lett 95, 207003 (2005).

20 O.K. Anderson, A.I. Liechtenstein, O. Jepson, and F. Paulsen, J. Phys. Chem. Solids 56, 1573 (1995).

21 A.A. Kordyuk, S.V. Borisenko, M. Knupfer, and J. Fink, Phys. Rev. B 67, 064504 (2003).

22 Note, $\chi(\mathbf{q}, \omega)$ results from the Green's function $\left\langle\left\langle S_{\mathbf{q}}^{+} \mid S_{-\mathbf{q}}^{-}\right\rangle\right\rangle$, where the spin operators are $S_{\mathbf{q}}^{+}=S_{1 \mathbf{q}_{||}^{+}}^{+i q_{z} \frac{d}{2}}+S_{2 \mathbf{q}_{\|}}^{+} e^{-i q_{z} \frac{d}{2}}$ and $S_{1}^{+}=S_{e}^{+}+S_{o}^{+}$, $S_{2}^{+}=S_{e}^{+}-S_{o}^{+}$. This transformation yields Eq.(5).

${ }^{23}$ For the numerical calculation of $\chi_{0}$, we employed $\delta=2$ $\mathrm{meV}$ in the analytic continuation of the Greens functions $i \omega_{n} \rightarrow \omega+i \delta$.

24 D.K. Morr and D. Pines, Phys. Rev. B 62, 15177 (2000); ibid. Phys. Rev. B 61, R6483 (2000).

25 M. Eschrig, and M.R. Norman, Phys. Rev. B 67, 144503 (2003).

26 T. Dahm, and I. Eremin, Phys. Rev. Lett. 97, (2006) (in press). 